\title{
The mRNA, miRNA and IncRNA networks in hepatocellular carcinoma: An integrative transcriptomic analysis from Gene Expression Omnibus
}

\author{
JIAN-HUA XU, WEI-HUA CHANG, HANG-WEI FU, TAO YUAN and PING CHEN \\ Department of Hepatobiliary Surgery, Daping Hospital and Research Institute of Surgery, \\ Third Military Medical University, Chongqing 400042, P.R. China
}

Received April 12, 2017; Accepted October 23, 2017

DOI: $10.3892 / \mathrm{mmr} .2018 .8694$

\begin{abstract}
Research advances and analysis in the non-protein coding part of the human genome have suggested that microRNAs (miRNAs) and long noncoding RNAs (lncRNAs) are associated with tumor initiation, growth and metastasis. Accumulating studies have demonstrated that a class of miRNAs and lncRNAs are dysregulated in hepatocellular carcinoma (HCC) and closely associated with tumorigenesis, diagnosis and prognosis. In the present study, integrative analysis of published data on multi-level Gene Expression Omnibus (GEO) and a bioinformatics computational approach were used to predict regulatory mechanism networks among differentially expressed mRNAs, miRNAs, and lncRNAs. Firstly, nine microarray expression data sets of mRNAs, miRNAs, and lncRNAs associated with HCC were collected from GEO datasets. Secondly, a total of 628 mRNAs, 15 miRNAs, and 49 lncRNAs were differentially expressed in this integrative analysis. Following this, mRNA, miRNA and IncRNA regulatory or co-expression networks were constructed. From the construction of the regulatory networks, five miRNAs and ten IncRNAs were identified as key differentially expressed noncoding RNAs associated with HCC progression. Finally, the regulatory effects of ten lncRNAs and miRNAs were validated. The study provides a novel insight into the understanding of the transcriptional regulation of HCC, and differentially expressed lncRNAs targeted and
\end{abstract}

Correspondence to: Professor Tao Yuan or Professor Ping Chen, Department of Hepatobiliary Surgery, Daping Hospital and Research Institute of Surgery, Third Military Medical University, 10 Changjiangzhilu Road, Yu Zhong, Chongqing 400042, P.R. China

E-mail: yuantaosyd@126.com

E-mail: chenpingsyd@163.com

Abbreviations: HCC, hepatocellular carcinoma; GEO, Gene Expression Omnibus; miRNA, microRNA; lncRNA, long non-coding RNA; KEGG, Kyoto Encyclopedia of Genes and Genomes

Key words: hepatocellular carcinoma, Gene Expression Omnibus, noncoding RNA, transcriptomic analysis, regulatory network regulated by miRNAs were identified and validated in HCC specimens and cell lines.

\section{Introduction}

Hepatocellular carcinoma (HCC) is the fifth most common cancer in men and the seventh in women, and the second or third most common cause of cancer-associated mortalities worldwide (1-3). However, the molecular pathogenesis of HCC is not fully understood. It is well known that during the process of tumor development, there are numerous levels of gene transcriptional regulation initiate and promote the process of tumor development and progression. Hence, improved understanding of the underlying molecular processes of tumor initiation and the identification of key molecular events for transcriptional regulation that promotes tumor initiation and growth are of great significance in developing therapeutic strategies.

Genome sequencing research has revealed that the human genome is comprised of $<2 \%$ protein-coding genes and $>90 \%$ of the genome is transcribed as non-coding RNA (ncRNA) (4). Noncoding RNAs, an example of which includes microRNAs (miRNAs), are non-coding RNAs which are 18-25 nucleotides in length, and long non-coding RNAs (lncRNAs) which are defined as transcripts containing $>200$ nucleotides that have a critical role in tumor occurrence and progression. It has been demonstrated that miRNAs are involved in every type of cancer examined to date, and the effects of miRNAs are mediated by binding to target mRNAs or IncRNAs, either to suppress mRNA translation or to degrade the miRNA-bound mRNA or lncRNA (5). Emerging evidence suggests that lncRNAs exhibit various critical roles in global gene regulation, including roles as the decoy, guide and in scaffolding $(6,7)$. It has previously been demonstrated that miRNAs and lncRNAs are involved in tumorigenesis, acting either as oncogenes or tumor suppressors in HCC (8). However, multiple avenues for feedback and interconnectivity among miRNAs, IncRNAs and mRNAs in HCC may potentially engender emergent cooperative behavior.

With the emergence of microarray technologies (9), the characterization of the mammal transcriptome may occur at an unprecedented resolution (10). The National Center for Biotechnology Information (NCBI) Gene Expression Omnibus (GEO; www.ncbi.nlm.nih.gov/geo/) provides the largest public 
repository of microarray data in existence $(11,12)$. Therefore, effective integration of HCC GEO datasets may lead to identification of differentially expressed lncRNAs, miRNAs and mRNAs at transcription level, which provide a better research means for tumor diagnosis, treatment and prognosis. There are numerous HCC-associated lncRNA, miRNA and mRNA microarray data; however, there are few studies on the integrative analysis of GEO datasets in transcriptional regulation associated with HCC. Therefore, understanding the potential regulation of lncRNAs, miRNAs and mRNAs expression is critical for revealing novel therapeutic targets and prognostic factors in management of $\operatorname{HCC}(13,14)$.

In the present study, differentially expressed lncRNAs, miRNAs and mRNAs that promote tumor initiation and growth were identified. The regulatory networks, between mRNA, miRNA and IncRNA were constructed, in addition to the co-expression network of mRNA-lncRNA. The key features of miRNAs regulatory effects on lncRNAs were annotated and validated in HCC specimens.

\section{Materials and methods}

Cell culture. The normal human hepatocyte cell line (L02) and human hepatocellular carcinoma cell lines (SMMC7721, Bel7404, Huh7 and PLC/PRF/5) were purchased from Cell Bank of Chinese Academy of Sciences (Shanghai, China) and maintained in Dulbecco's modified Eagle's medium (Invitrogen; Thermo Fisher Scientific, Inc., Waltham, MA, USA) supplemented with $10 \%$ fetal bovine serum (Invitrogen; Thermo Fisher Scientific, Inc.), $100 \mathrm{U} / \mathrm{ml}$ penicillin, $100 \mu \mathrm{g} / \mathrm{ml}$ streptomycin, and then cultured at $37^{\circ} \mathrm{C}$ in a humidified atmosphere containing $5 \% \mathrm{CO}_{2}$

Patients and specimens. The experimental protocol was approved by the Institutional Ethics Review Board of Daping Hospital, the Third Military Medical University. Written informed consent was obtained from all participants. A total of 10 patients with $\mathrm{HCC}$ were recruited randomly at inpatient service of the Department of Hepatobiliary Surgery, the Third Military Medical University between 2013 and 2014. Patients underwent surgical HCC resection; HCC and corresponding non-tumor liver tissues were collected. All specimens were snap-frozen in liquid nitrogen or stored at $-80^{\circ} \mathrm{C}$. The crystal HCC and non-tumor tissue sections $(4 \mu \mathrm{m})$ were stained with H\&E: Sections were mounted on glass slides, fixed with fixative (4\% paraformaldehyde) for $20 \mathrm{~min}$ at room temperature, air-dried for $15 \mathrm{~min}$, stained in hematoxylin solution (Beyotime Institute of Biotechnology, Haimen, China) for $2 \mathrm{~min}$ at $42^{\circ} \mathrm{C}$, then washed in running distilled water for $10 \mathrm{~min}$. Subsequently, sections were stained in $0.5 \%$ eosin solution (Beyotime Institute of Biotechnology) for $1 \mathrm{~min}$ at $42^{\circ} \mathrm{C}$, and then washed again, dehydrated, and mounted at room temperature to ensure homogenous cell population of tissues. Individual patients were excluded if they received any chemotherapy and radiotherapy. Among those 10 patients, 7 were male and 3 were female. The age ranged from $35-72$ years; 8 patients were serum positive for hepatitis B surface antigen.

Selection of GEO datasets, processing and construction of networks. The NCBI GEO (www.ncbi.nlm.nih.gov/geo) (11) was searched for expression profiling studies on mRNAs, miRNAs and lncRNAs in HCC. Explicit search strategies for three types of GEO datasets were applied to preliminary selection. The strategy for mRNA datasets, [((()liver cancer (Title)) OR Liver Neoplasms (MeSH Terms)) OR hepatocellular carcinoma (Title)) AND Homo sapiens (Organism))] AND [(mRNA) OR gene expression); for miRNA, (liver cancer (Title)) OR Liver Neoplasms (MeSH Terms)) OR hepatocellular carcinoma (Title)) AND Homo sapiens (Organism))] AND miRNA; for lncRNA, [((((liver cancer (Title)) OR Liver Neoplasms (MeSH Terms)) OR hepatocellular carcinoma (Title)) AND Homo sapiens (Organism))] AND lncRNA, with a time limit until August 2015. The inclusion and exclusion criteria included GEO datasets of RNA transcriptome analysis of human HCC tissues from microarray in published articles; GEO datasets from non-HCC tissue samples were excluded. A total of nine sets of GEO microarray data were identified from the initial literature search and manual search (Table I). According to NCBI probe annotation files, gene expression levels (average levels of the corresponding probes) were calculated. For lncRNA genes, expression levels were calculated according to human transcript sequences (refseq version) downloaded from UCSC (www.genome.ucsc.edu/) (15) and lncRNAs probe sequence annotation from NONCODE V4 (16). Differentially expressed genes were screened through a matrix of gene expression levels with the bioconductor limma R package (17). The threshold of differentially expressed genes screened was $\mathrm{P}<0.05$, Ifold changel $>1.5$. Data were integrated according to the results of the differentially expressed genes in the final screening. The standards of screening differentially expressed genes suggest that genes must be present and differentially expressed in at least two datasets, and that the trend of expression alteration is consistent. The target mRNA of miRNA was screened from microT-CDS (18), miRanda (19), miRDB (20), PITA (21), TargetScan 6.2 (22), miRWalk (23) and miRecords (24), where miRWalk and miRecords are included in the validated target genes. Screening for the miRNA target gene involved the miRNA and the target gene appearing in the validation database or in at least three prediction databases, and the miRNAs and the target genes exhibiting differing expression levels, with opposite expression alteration trends. The regulatory network of miRNAs-mRNAs was constructed using Cytoscape software (25). In addition, the target genes for each miRNA were analyzed by Kyoto Encyclopedia of Genes and Genomes (KEGG) (26) enrichment $(\mathrm{P}<0.05$, and at least two genes in the pathway). The expression levels of lncRNA and mRNA in the three GEO datasets (GSE58043, GSE55191, GSE27462) (27-29) were first integrated, and then screened according to their expression correlation (Pearson correlation), and screened with IPearson correlation coefficient $\geq 0.7$. The co-expression network of mRNAs-lncRNAs was constructed using Cytoscape software (30) and the co-expressed mRNAs were analyzed for KEGG enrichment analysis of each lncRNA. According to the miRNA sequence and IncRNA sequence of NONCODE V4, miRanda software was used to predict the interaction between miRNAs and lncRNAs. The associated pairs were screened according to the miRNA and lncRNA differing expressions and opposite 
Table I. Selected GEO datasets.

\begin{tabular}{lccc}
\hline Microarray types & GEO accession & Number of tumor samples & Number of adjacent non-tumor samples \\
\hline mRNA microarray & GSE25097 & 268 & 243 \\
& GSE57957 & 39 & 39 \\
miRNA microarray & GSE22405 & 24 & 24 \\
& GSE31384 & 166 & 166 \\
\multirow{5}{*}{ lncRNA microarray } & GSE36915 & 68 & 21 \\
& GSE10694 & 78 & 78 \\
& GSE58043 & 7 & 7 \\
& GSE55191 & 3 & 3 \\
\hline
\end{tabular}

GEO, Gene Expression Omnibus; lncRNA, long non-coding RNA; miRNA, microRNA.

Table II. Mimics of miRNAs

\begin{tabular}{lll}
\hline miRNA mimic & \multicolumn{1}{c}{ Sense (5'-3') } & \multicolumn{1}{c}{ Anti-sense (5'-3') } \\
\hline Hsa-miR-101-3p mimic (miR-101) & UACAGUACUGUGAUAACUGAA & CAGUUAUCACAGUACUGUAUU \\
Hsa-miR-125b-5p mimic (miR-125b) & UCCCUGAGACCCUAACUUGUGA & ACAAGUUAGGGUCUCAGGGAUU \\
Hsa-miR-130a-3p mimic (miR-130a) & CAGUGCAAUGUUAAAAGGGCAU & CCCUUUUAACAUUGCACUGUU \\
Hsa-miR-195-5p mimic (miR-195) & UAGCAGCACAGAAAUAUUGGC & CAAUAUUUCUGUGCUGCUAUU \\
Hsa-miR-145-3p mimic (miR-145) & GGAUUCCUGGAAAUACUGUUCU & AACAGUAUUUCCAGGAAUCCUU \\
Negative control mimic & UCACAACCUCCUAGAAAGAGUAGA & UACUCUUUCUAGGAGGUUGUGAUU
\end{tabular}

miR, miRNA, microRNA.

trends, and the networks were constructed using Cytoscape software.

Transfection of miRNA mimics. Cells were seeded in 6-well plates at a concentration of $2 \times 10^{5}$ cells/well. When cells reached $40-60 \%$ confluence, $150 \mathrm{nM}$ miRNA mimics (Guangzhou RiboBio Co., Ltd., Guangzhou, China), and negative control (NC) miRNA mimics were transfected using Lipofectamine ${ }^{\circledR} 2000$ reagent (Invitrogen; Thermo Fisher Scientific, Inc.) according to the manufacturer's protocol, for $24 \mathrm{~h}$. The miRNA mimics and NC mimics were synthesized by Guangzhou RiboBio Co. Ltd. and sequences are listed in Table II.

Reverse transcription-quantitative polymerase chain reaction $(R T-q P C R)$. Total RNA was extracted from tissue samples and cells using TRIzol ${ }^{\circledR}$ reagent (Invitrogen; Thermo Fisher Scientific, Inc.) according to the manufacturer's protocols. The total RNA was quantified by using a Nanodrop ND-1000 spectrophotometer (NanoDrop Technologies; Thermo Fisher Scientific, Inc.), and one microgram of RNA was used for cDNA synthesis using GeneAmp RNA PCR kit (Applied Biosystems; Thermo Fisher Scientific, Inc.). Gene expression was examined by SYBR-Green PCR Master mix (Applied Biosystems; Thermo Fisher Scientific, Inc.) using the primers (as listed in Table III) with Bio-Rad CFX96 qPCR system. The detection run started at $50^{\circ} \mathrm{C}$ for $2 \mathrm{~min}, 95^{\circ} \mathrm{C}$ for $2 \mathrm{~min}$, followed by 45 cycles at $95^{\circ} \mathrm{C}$ for $15 \mathrm{sec}$ and at $60^{\circ} \mathrm{C}$ for $1 \mathrm{~min}$. The data were analyzed using the $2^{\Delta \mathrm{Ct}}$ method or $2^{-\Delta \Delta \mathrm{Cq}}$ method (31). The lncRNA expression levels were normalized to the GAPDH level.

Statistical analysis. Data are present as the mean \pm standard error of the mean. The statistical significance between the experimental groups was assessed using one-way analysis of variance, unpaired Student's t-test or non-parametric test. Statistical analysis and curve fitting were performed using GraphPad Prism software 5.1 (GraphPad Software, Inc., San Diego, CA, USA) and SPSS 19.0 for Windows (IBM-SPSS, Chicago, IL, USA). $\mathrm{P}<0.05$ was considered to indicate a statistically significant difference.

\section{Results}

Identification of differentially expressed mRNAs, miRNAs and lncRNAs. Microarray expression data of HCC associated mRNAs, miRNAs, and lncRNAs were collected from GEO: This consisted of a total of 3 mRNA expression datasets, including 331 samples of tumor tissue and 306 samples of adjacent non-tumor tissues, 3 miRNA expression datasets, including 312 samples of tumor tissue and 265 samples of adjacent non-tumor tissue, and 3 lncRNA expression datasets, including 15 samples of tumor tissue and 15 samples of adjacent non-tumor tissue (Table I). The results demonstrated that 
Table III. Sequences of long non-coding RNA primers for reverse transcription-quantitative polymerase chain reaction assays.

\begin{tabular}{|c|c|c|c|}
\hline Name & Primer sequence & Annealing temperature & Product length (bp) \\
\hline \multicolumn{4}{|c|}{ NONHSAG048960 (NON960) ${ }^{\mathrm{a}}$} \\
\hline $\mathrm{F}$ & TTCAGGAGACACGCGGACTA & 59 & 294 \\
\hline $\mathrm{R}$ & GCTAATCTGGGTCAGGAGCG & & \\
\hline \multicolumn{4}{|c|}{ NONHSAG041512 (NON512) } \\
\hline $\mathrm{F}$ & TTAGGATAGGATGGGCTTTTTCTGT & 60 & 101 \\
\hline $\mathrm{R}$ & AATTGCCCTAGACCCAGTGGT & & \\
\hline \multicolumn{4}{|c|}{ NONHSAG016418 (NON418) } \\
\hline $\mathrm{F}$ & AACTGGGCTTCCGTAGAACG & 60 & 242 \\
\hline $\mathrm{R}$ & GAAGGGGTGTAACGGGCAAA & & \\
\hline \multicolumn{4}{|c|}{ NONHSAG028411 (NON411) } \\
\hline $\mathrm{F}$ & СTCAATGGCCTGGGAGGTTT & 59 & 160 \\
\hline $\mathrm{R}$ & ACAAGTTCTGTGAGGGCAGG & & \\
\hline \multicolumn{4}{|c|}{ NONHSAG020621 (NON621) } \\
\hline $\mathrm{F}$ & TCTGGTGGACCCAACTCTGT & 60 & 153 \\
\hline $\mathrm{R}$ & CTTTGTCTTAGGCCAGCGGT & & \\
\hline \multicolumn{4}{|c|}{ NONHSAG012658 (NON658) } \\
\hline $\mathrm{F}$ & AGCTTAGTCGCTCATCTGGC & 61 & 226 \\
\hline $\mathrm{R}$ & AGTCAGCCAGTTCGGAAACC & & \\
\hline \multicolumn{4}{|c|}{ NONHSAG019946 (NON946) } \\
\hline $\mathrm{F}$ & СCСАТАСТТССССТТССАGC & 60 & 122 \\
\hline $\mathrm{R}$ & ATTGCAGTTGGGCAGAGTGA & & \\
\hline \multicolumn{4}{|c|}{ NONHSAG034094 (NON094) } \\
\hline $\mathrm{F}$ & GTCGTGTCTCCTTCTTGGGG & 60 & 109 \\
\hline $\mathrm{R}$ & AGCGGTCATTATCTAGCGCC & & \\
\hline \multicolumn{4}{|c|}{ NONHSAG048615 (NON615) } \\
\hline $\mathrm{F}$ & CCCTACAAGTGGCTTTCGTG & 60 & 327 \\
\hline $\mathrm{R}$ & CGGACCCCAGAATACACCAC & & \\
\hline \multicolumn{4}{|c|}{ NONHSAG006679 (NON679) } \\
\hline $\mathrm{F}$ & TGTCTGATTCTGTCTGCTCCA & 61 & 172 \\
\hline $\mathrm{R}$ & CCGCATTTTCCCCATTCCAG & & \\
\hline \multicolumn{4}{|c|}{ NONHSAG051177 (NON679) } \\
\hline $\mathrm{F}$ & CTGCAAGTTTTGACCACGTCC & 60 & 94 \\
\hline $\mathrm{R}$ & AGACAATGAACAGGGCACAGAT & & \\
\hline \multicolumn{4}{|c|}{ NONHSAG001301 (NON301) } \\
\hline $\mathrm{F}$ & CCACAGTCCCGCTTACTTGT & 60 & 263 \\
\hline $\mathrm{R}$ & TTAAACCCGAGGGGGAGGAT & & \\
\hline \multicolumn{4}{|c|}{ GAPDH } \\
\hline $\mathrm{F}$ & TGCACCACCAACTGCTTAGC & 58 & 87 \\
\hline $\mathrm{R}$ & GGCATGGACTGTGGTCATGAG & & \\
\hline
\end{tabular}

${ }^{\mathrm{a} N O N C O D E}$ ID, shorter form of NONCODE ID; F, forward; R, reverse.

628 mRNAs, 15 miRNAs, and 49 lncRNAs were significantly differentially expressed in HCC.

Construction of regulatory networks and co-expression network. The miRNA-mRNA regulatory network was constructed with a total of 87 pairs of upregulated miRNAs and downregulated target mRNAs (Fig. 1A), 255 association pairs of downregulated miRNAs and upregulated target mRNAs (Fig. 1B). According to the results of the enrichment analysis (Fig. 2), the target genes of hsa-miR-222-3p and hsa-miR-195-5p were enriched in cancer associated pathways, hsa-miR-224-5p in p53 associated pathways, and hsa-miR-125b-5p in the pathway of antigen presentation and immune correlation. Previous studies additionally demonstrated 

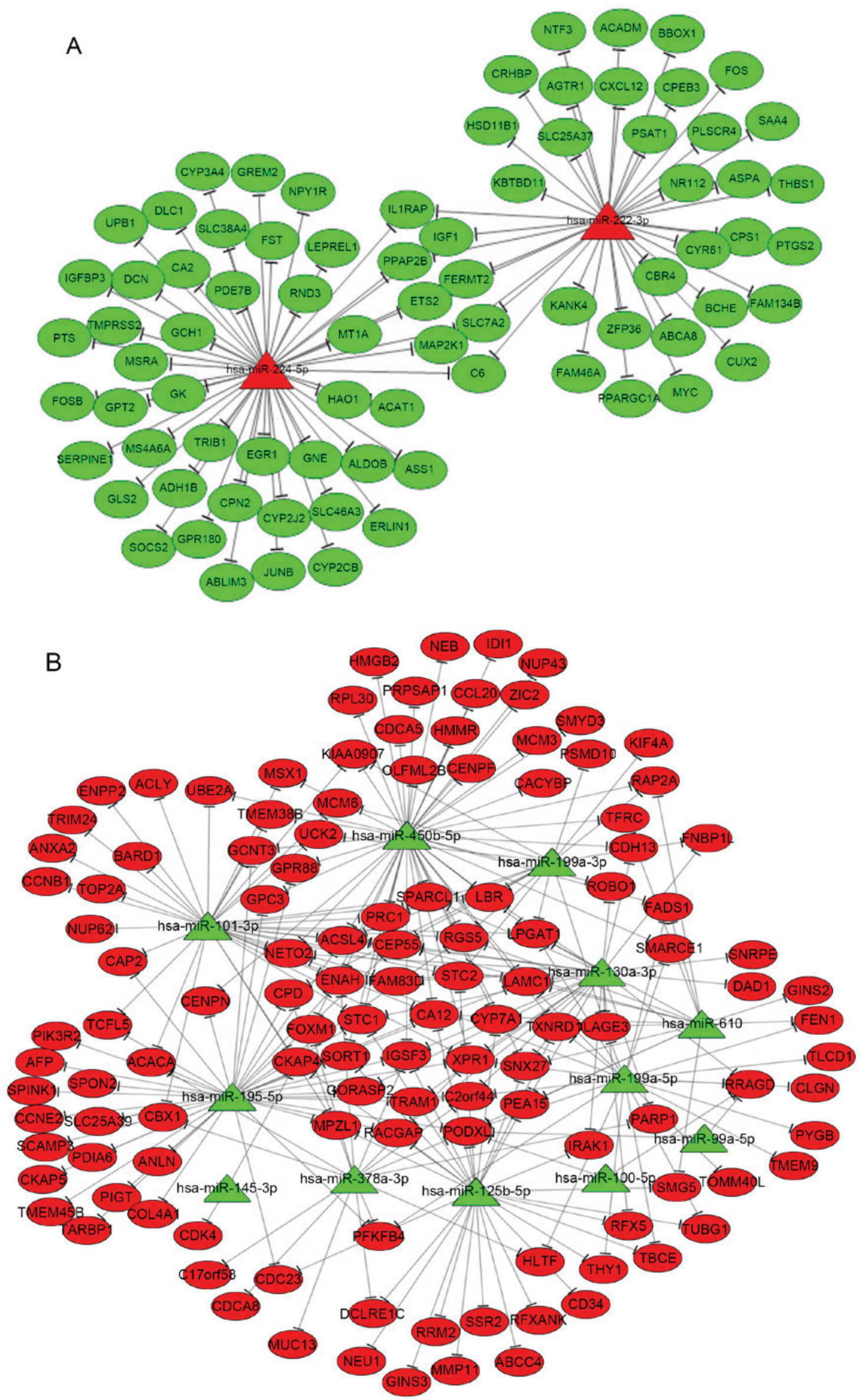

Figure 1. Construction of regulatory networks between miRNAs and mRNAs. (A) Association between upregulated miRNAs and downregulated target mRNAs in the network. (B) Association of downregulated miRNAs and upregulated target mRNAs in the network. Circles indicate mRNA and triangles indicate miRNA. Red represents high expression and green represents low expression. miRNA, microRNA. 


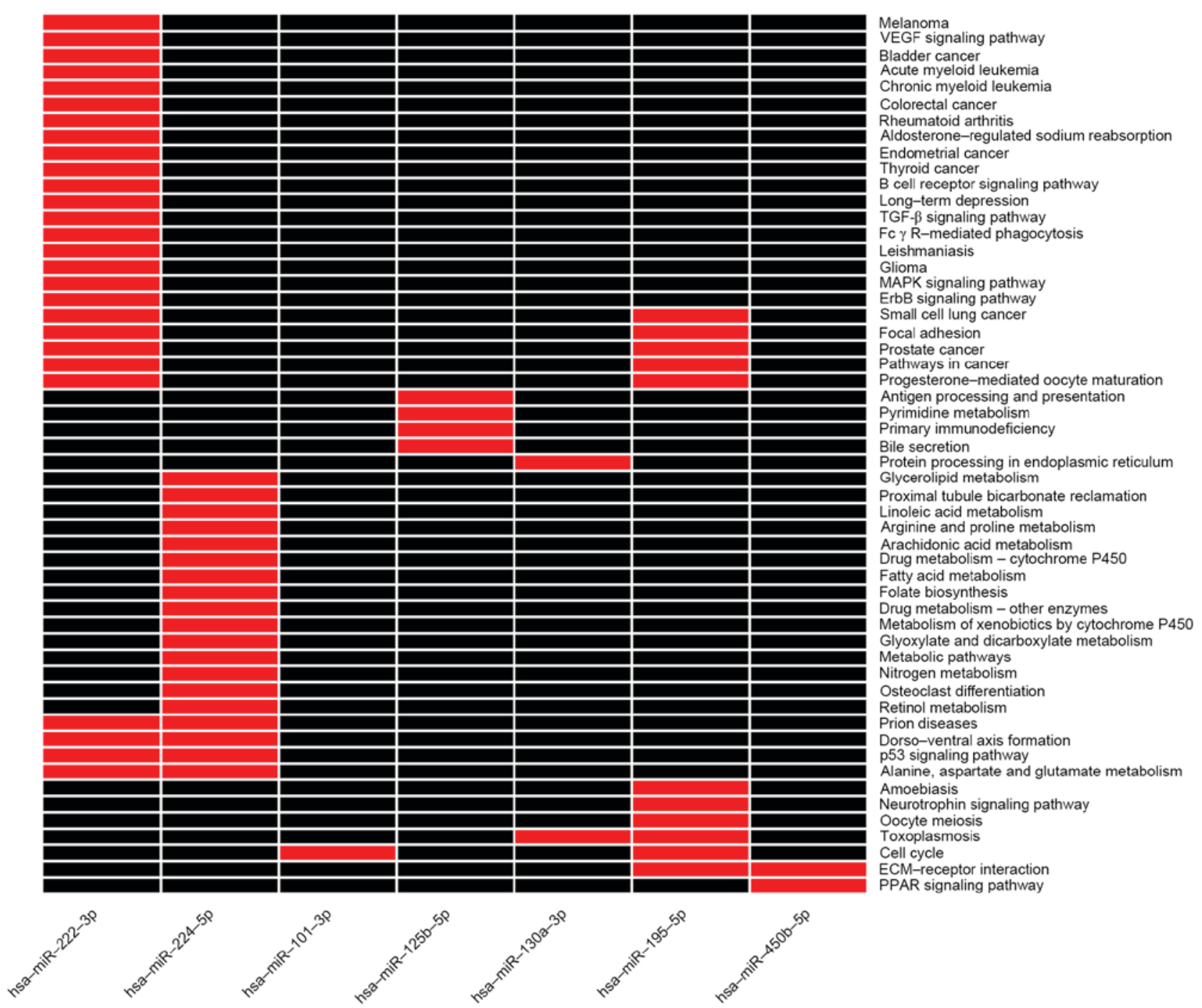

Figure 2. Annotation and Kyoto Encyclopedia of Genes and Genomes enrichment pathway analysis of differentially expressed miRNAs. Red represents the pathway of enrichment by miRNAs targeting mRNAs. $\mathrm{P}<0.05$, and at least two genes are in the pathway. miRNA, microRNA.

that hsa-miR-222-3p (32-34), hsa-miR-125-5p (35), hsa-miR-224-5p (34) and hsa-miR-195 $(36,37)$ are involved in the critical processes of tumorigenesis. In addition, differentially expressed mRNAs exhibit an important role in HCC tumorigenesis, including a-fetoprotein $(38,39)$, glypican 3 (40,41), and forkhead box M1 (42). Fig. 3 presents the co-expression network of mRNAs-lncRNAs. The enrichment results of mRNA KEGG co-expressed with lncRNA are presented in Fig. 4, in which the majority of lncRNAs co-expressed with mRNAs were enriched in the cell cycle pathway. It was revealed that NONHSAG046766, NONHSAG011461 and NONHSAG016418 were enriched in the p53 pathway. According to the results of miRNA-regulated IncRNAs predicted by miRanda, combined with the significantly differentially expressed miRNAs and lncRNAs, the miRNA-lncRNA regulatory network was constructed with a total of 110 miRNA-lncRNA association pairs screened (Fig. 5). According to the number of connections per miRNA and lncRNA (as node) and the KEGG results, 5 miRNAs and 10 lncRNAs were selected as key differentially expressed noncoding RNAs in integrative analysis (presented in the blue circle).
Expression of lncRNAs in cell lines and HCC tissues. To validate the findings of the integrated microarray analysis, transcripts of 10 key differentially expressed lncRNAs, which were upregulated in integrated analysis, were analyzed by RT-qPCR in 5 cell lines and 10 pairs of randomly selected, paired tumor and non-tumor liver tissues from $10 \mathrm{HCC}$ patients. The present study primarily focused on predicted upregulated lncRNAs, as lncRNAs may be more readily used as early diagnosis markers or therapeutic targets, compared with downregulated IncRNAs. The RT-qPCR analysis verified the findings of integrated microarray analysis. Compared with the human normal liver cell line (L02) and human non-tumor adjacent tissues, the 10 lncRNA expression levels were increased in cancer cell lines (Fig. 6A) and tumor tissues (Fig. 6B).

Validation of miRNA-lncRNA regulatory associations in vitro. Interactions between IncRNAs and miRNAs, which are important classes of noncoding RNAs in eukaryotes, provide an additional layer of control in gene regulation. The 5 miRNAs and 10 lncRNAs in core miRNAs-lncRNAs regulatory network were selected to validate miRNAs regulatory 


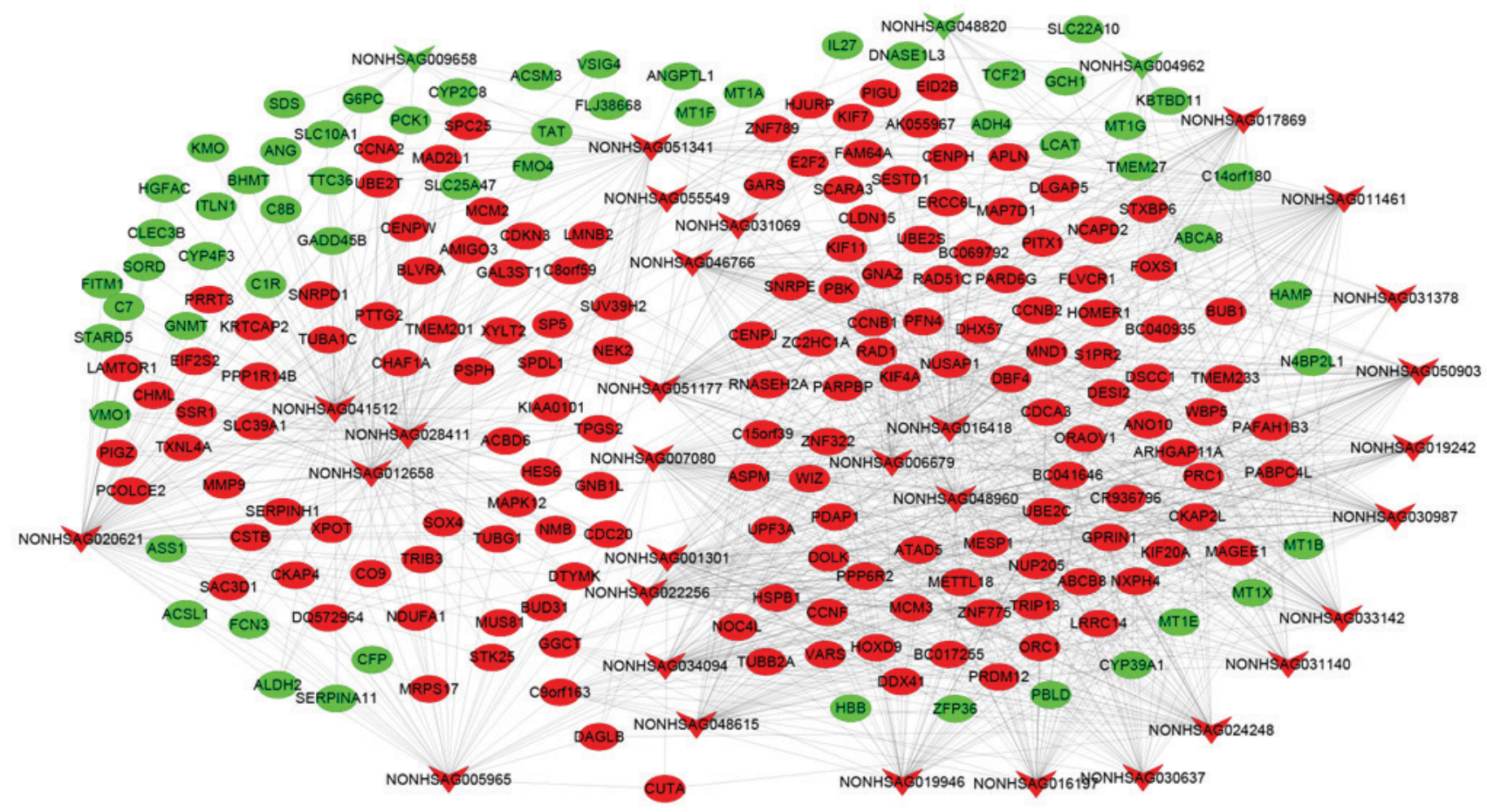

Figure 3. Construction of lncRNA-mRNA co-expression networks. LncRNA-mRNA co-expression networks of all differentially expressed lncRNAs and mRNAs with IPearson correlation coefficient I 0.7. Circles indicate mRNA and V shape indicates lncRNA. Red represents high expression and green represents low expression. LncRNA; lncRNA, long non-coding RNA.

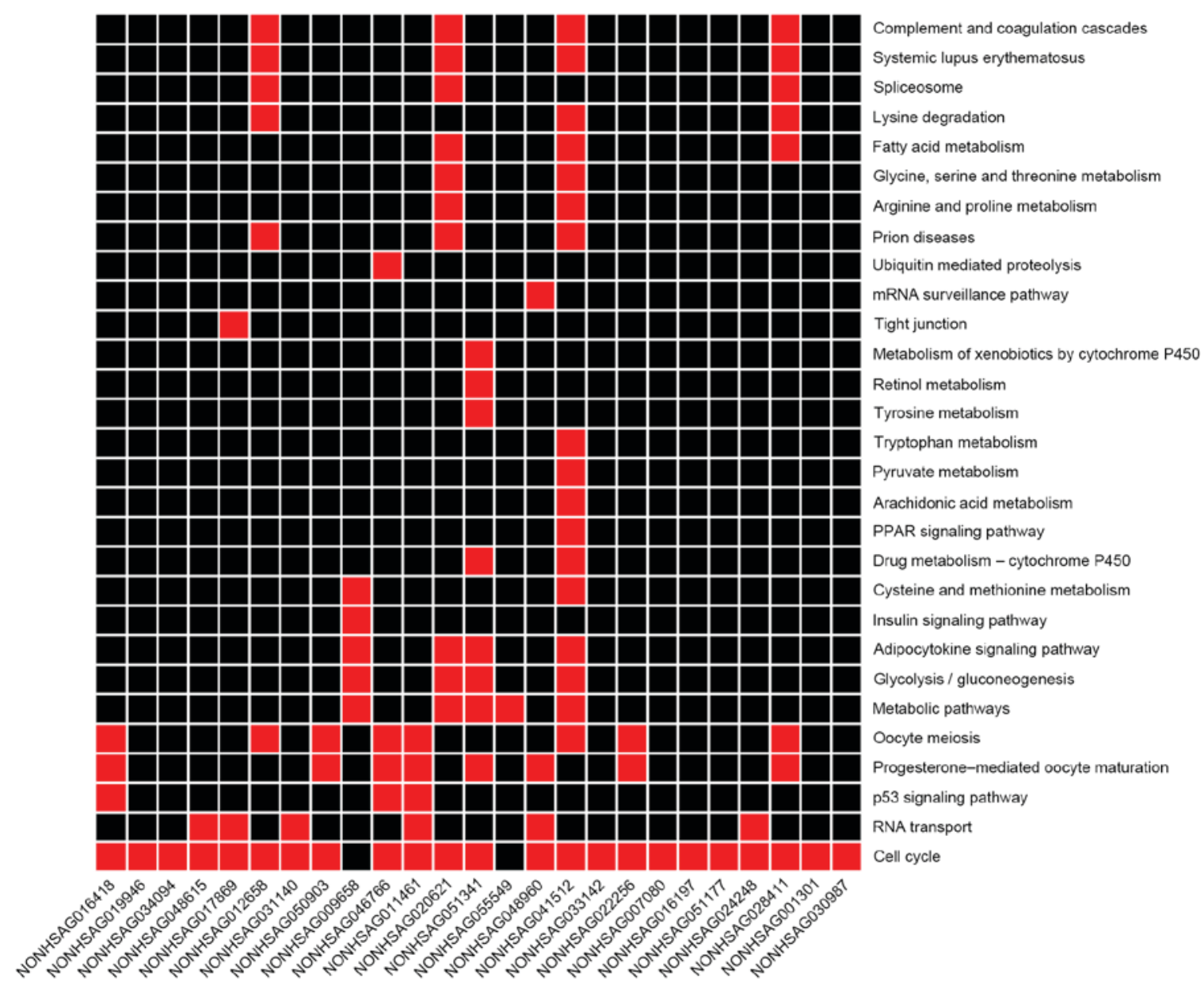

Figure 4. Annotation and Kyoto Encyclopedia of Genes and Genomes enrichment pathway analysis of differentially expressed lncRNAs. The majority of IncRNAs co-expressed with mRNAs were enriched in the cell cycle pathway. Red represents the pathway of enrichment by lncRNAs co-expressed with mRNAs. $\mathrm{P}<0.05$, and at least two genes are in the pathway. LncRNA; IncRNA, long non-coding RNA. 


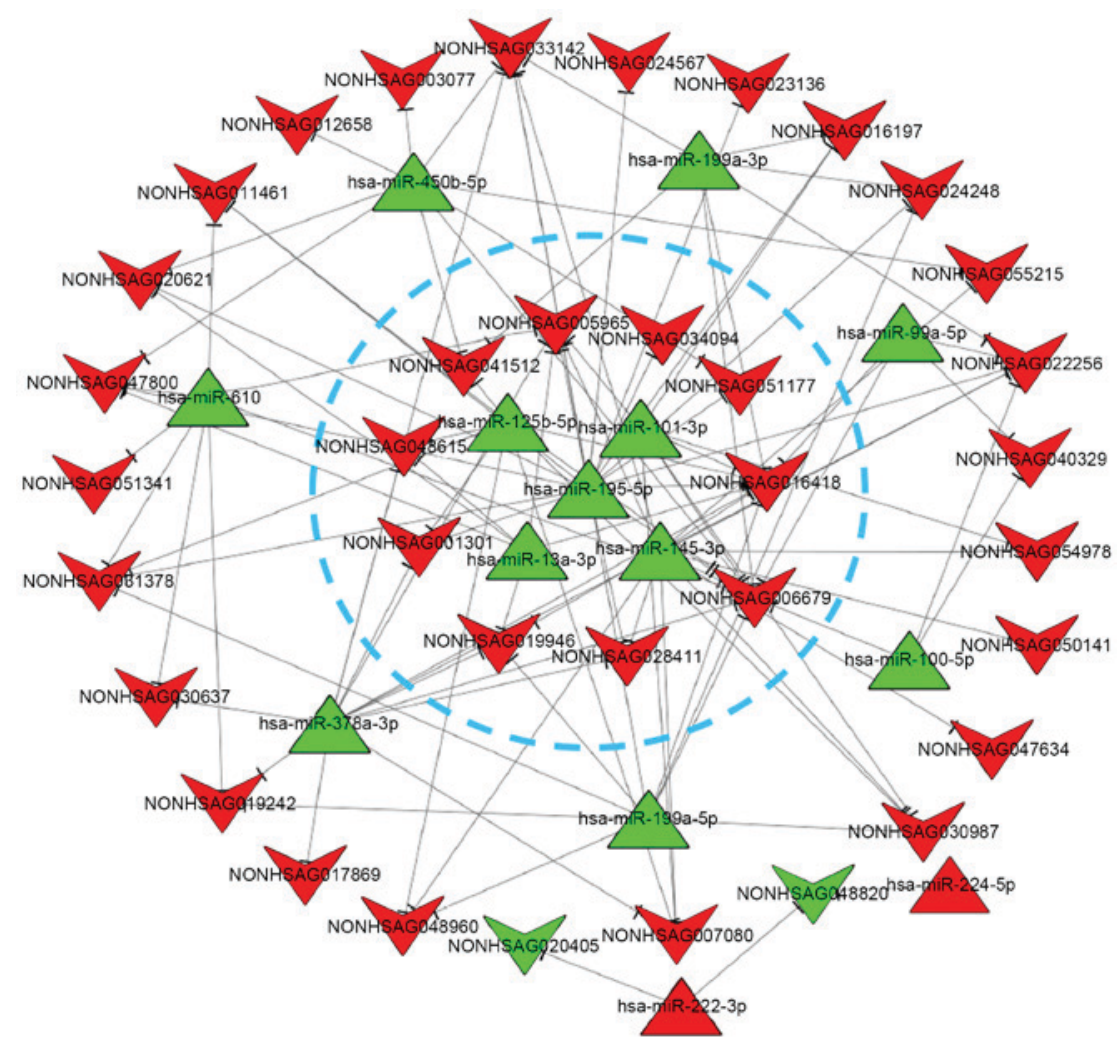

Figure 5. Construction of miRNA-lncRNA association networks. Association networks of all differentially expressed miRNAs and lncRNAs predicted by miRanda software. The 5 miRNAs and 10 lncRNAs in the blue circle represent the core network. V shape indicates lncRNA and triangle indicates miRNA. Red represents high expression and green represents low expression. miRNA, microRNA; lncRNA, long non-coding RNA.

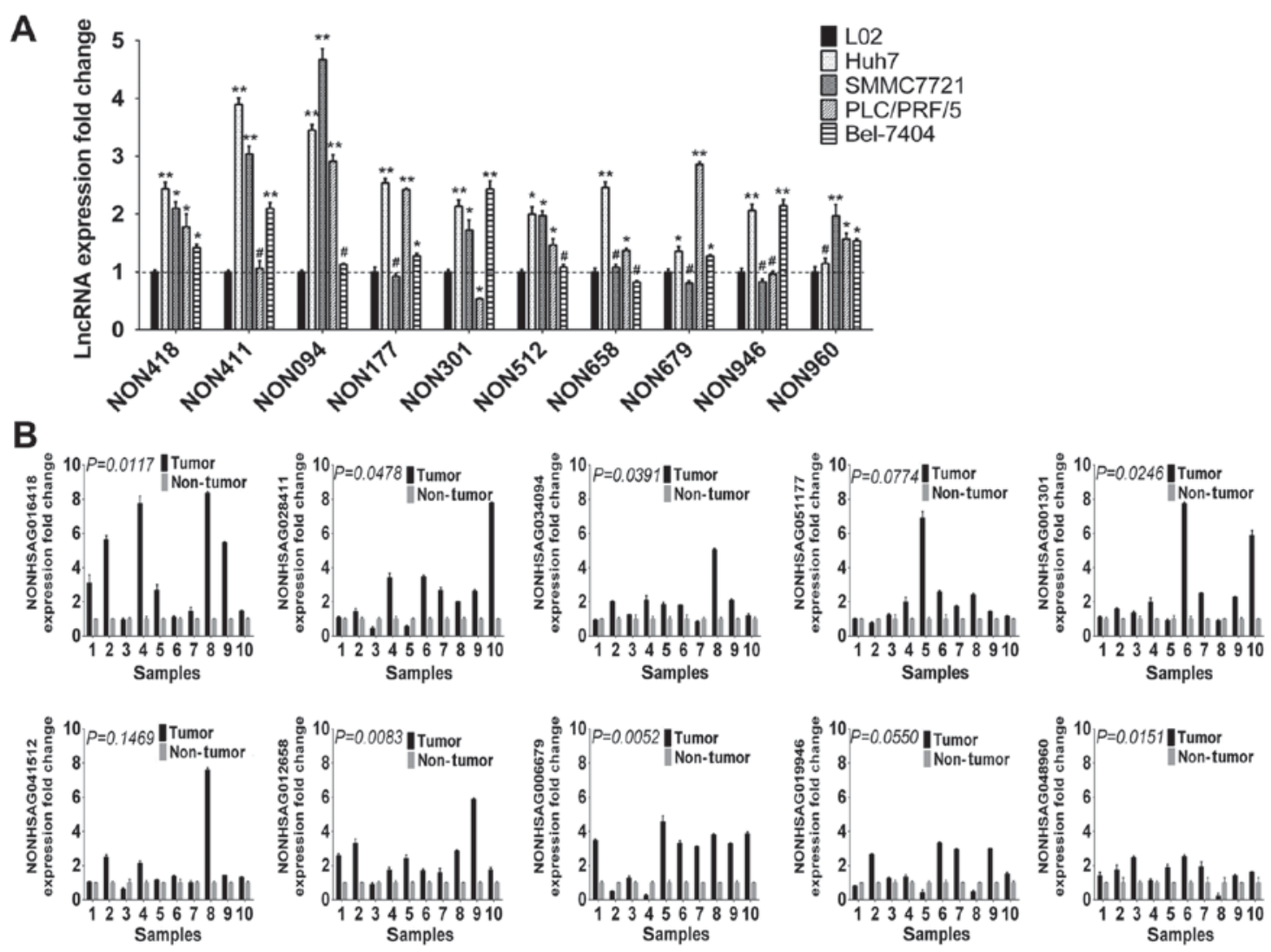

Figure 6. Expression of 10 lncRNAs by reverse transcription-quantitative polymerase chain reaction in 5 cell lines and 10 HCC tissues. (A) Expression of 10 lncRNAs in 5 cell lines. The expression level was normalized against GAPDH. Expression levels of lncRNAs in the L02 cell line was used as control group. (B) The levels of 10 lncRNAs in HCC tissues. The expression levels were normalized against GAPDH. Data are presented as the mean \pm standard error of the mean of three separate experiments. The levels of lncRNAs in adjacent non-tumor tissues were used as the controls. ${ }^{*} \mathrm{P}<0.05,{ }^{* * *} \mathrm{P}<0.01$ and ${ }^{*} \mathrm{P}>0.05$ vs. the controls. 

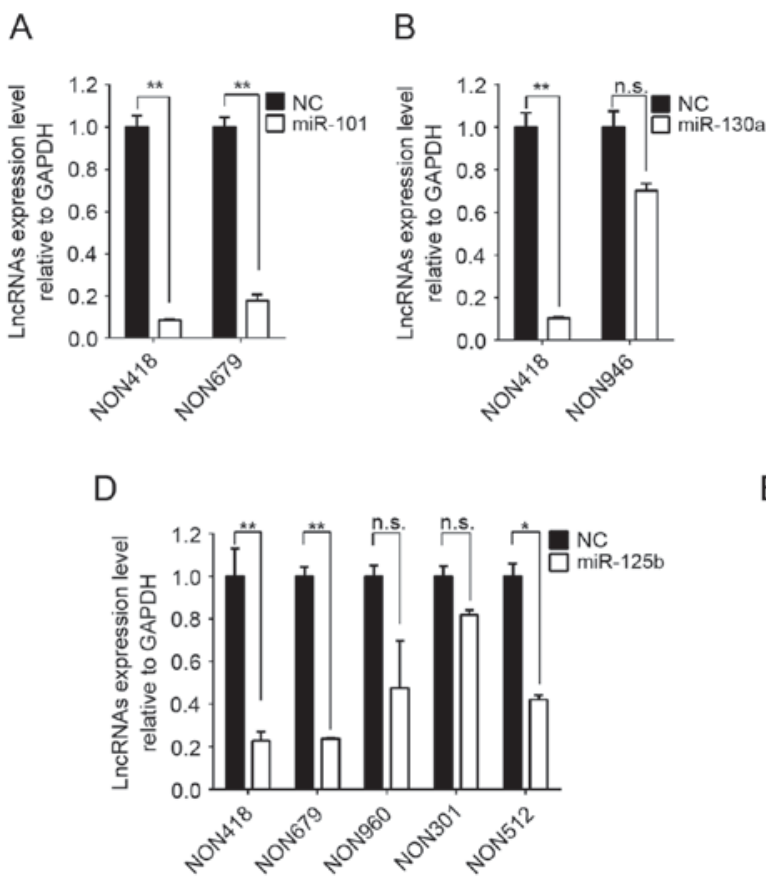

Figure 7. Validation of miRNAs regulatory effect on lncRNAs in vitro. The expression of each predicted lncRNA was detected by reverse transcription-quantitative polymerase chain reaction assay, following transfection of miRNA (A) hsa-miR-101-3p, (B) hsa-miR-130a-3p, (C) hsa-miR-145-3p, (D) hsa-miR-125b-5p and (E) hsa-miR-195-5p mimics for $24 \mathrm{~h}$. The expression level was normalized against GAPDH. Data are presented as the mean \pm standard error of the mean of three independent experiments. ${ }^{*} \mathrm{P}<0.05,{ }^{* *} \mathrm{P}<0.01$ vs. NC group. NC, negative control; ns, not significant; lncRNA, long non-coding RNA; miRNA, microRNA.

effect on lncRNAs. Following transfection of miRNAs, the majority of the upregulated lncRNAs altered their expression levels as predicted, and were downregulated compared with negative control (NC) (Fig. 7).

\section{Discussion}

Hepatocellular carcinoma remains a primary challenge due to its high morbidity and mortality without early diagnosis and lack of effective treatment. A few molecular biomarkers have been successfully used in clinical diagnostics, particularly as prognostic or diagnostic tools, and even as therapeutic targets for HCC. LncRNAs were once considered as the transcription noise, however, in-depth studies, along with a large number of clinical observations and experimental studies, have revealed that lncRNAs exhibit an important role in tumorigenesis and cancer development by interacting with miRNAs, mRNAs, and even proteins. Increasing evidence suggests that dysregulated lncRNAs are closely associated with the initiation and progression of HCC. Long intergenic non-coding RNA LINC00152 functions in gastric cancer (43); highly expressed long intergenic noncoding RNA UFC1 interacts with the mRNA stabilizing protein HuR to increase levels of $\beta$-catenin in HCC cells (44); growth arrest-specific 5 regulates apoptosis in prostate cancer (45). The results of these studies suggest that there are numerous novel lncRNAs that remain to be identified and investigated.

The present study systematically analyzed the complex effects of interrelated mRNAs, miRNAs and IncRNAs to provide networks for revealing the dysregulated lncRNAs. Currently, there are numerous investigations regarding mRNAs and miRNAs, however, research on IncRNAs remains limited, and the functions and mechanism of numerous lncRNAs remain
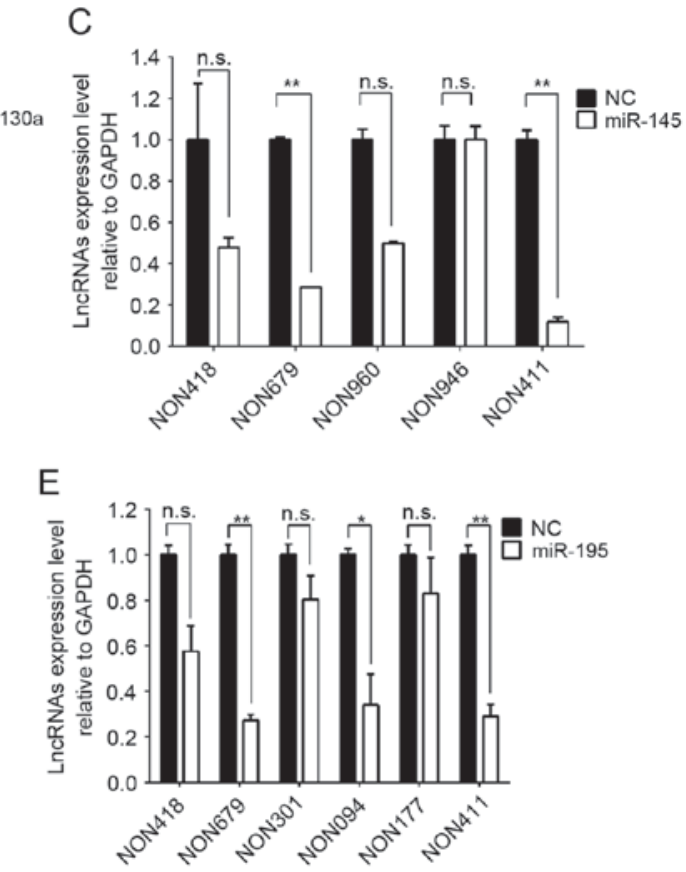

to be elucidated. The present study integrated GEO expression microarrays to identify differentially expressed mRNAs, miRNAs and lncRNAs, and further constructed networks to reveal the potential function and regulation mechanisms of the identified dysregulated lncRNAs. Consistent with the predicted results, the majority of IncRNAs were significantly differentially expressed in hepatocellular carcinoma cells and tissues. The results revealed that a particular set of miRNAs and IncRNAs were potentially involved in regulative mechanisms in HCC development at the transcription level. Previous molecular biology research conducted on the interaction between miRNAs and lncRNAs demonstrates that lncRNAs may be regulated by miRNAs. Cao et al (27) discovered that miR-34a targets and regulates linRNA UFC1 in HCC cells, $\mathrm{Xu}$ et al (45) reported that IncRNA-AC130710 is targeted by miR-129-5p in gastric cancer, and in addition, lncRNA MEG3 may be regulated by miR-29 in HCC (46). These studies indicated that lncRNAs may be targeted and regulated by miRNAs in the process of tumor genesis and evolution.

However, the present study had various limitations that should be acknowledged. The first is the shortage of expression microarray data for HCC in public GEO datasets. Results in the present study were primarily obtained through integrative analysis of the GEO database, and numerous cases included in the GEO microarray and analysis platform were not uniform. The integrative analysis was primarily based on the differentially expressed genes of the GEO microarray. Secondly, it should be emphasized that the regulatory networks or mechanisms analyzed in the study were only bioinformatically predicted, and expressions of a few lncRNAs were verified in cell lines and patients. In the future, further validation and functional examination of. miRNAs-IncRNAs may be conducted in vivo and in vitro. 
In conclusion, the present integrative analysis of the GEO transcriptomic data provided a comprehensive meaningful insight into the tumorigenesis of HCC and an understanding of the underlying mRNA-miRNA-lncRNA molecular mechanisms involved. The present study demonstrated a method to identify a novel class of potential biomarkers in HCC development. These findings indicated that upregulated lncRNAs, downregulated by miRNAs, may serve as potential molecular targets for the development of specific therapies for HCC.

\section{Acknowledgements}

The authors would like to thank Miss Jia Wang (research assistant) of the Department of Environmental Hygiene, College of Preventive Medicine, Third Military Medical University (Chongqing, China) for the critical reading of the manuscript. The present study was supported by the National Natural Science Foundation of China (grant no. 81270523).

\section{References}

1. El-Serag HB and Rudolph KL: Hepatocellular carcinoma: Epidemiology and molecular carcinogenesis. Gastroenterology 132: 2557-2576, 2007.

2. Bosetti C, Turati F and La Vecchia C: Hepatocellular carcinoma epidemiology. Best Pract Res Clin Gastroenterol 28: 753-770, 2014.

3. Wallace MC, Preen D, Jeffrey GP and Adams LA: The evolving epidemiology of hepatocellular carcinoma: A global perspective. Expert Revi Gastroenterol Hepatol 9: 765-779, 2015.

4. Shi X, Sun M, Liu H, Yao Y and Song Y: Long non-coding RNAs: A new frontier in the study of human diseases. Cancer Lett 339: 159-166, 2013.

5. Zhao T, Xu J, Liu L, Bai J, Wang L, Xiao Y, Li X and Zhang L: Computational identification of epigenetically regulated lncRNAs and their associated genes based on integrating genomic data. FEBS Lett 589: 521-531, 2015.

6. Shahandeh A: Molecular mechanisms of oncogenic long non-coding RNAs. Biosci Res 10: 38-54, 2013.

7. Marchese FP and Huarte M: Long non-coding RNAs and chromatin modifiers: Their place in the epigenetic code. Epigenetics 9: 21-26, 2014.

8. Yang G, Lu X and Yuan L: LncRNA: A link between RNA and cancer. Biochim Biophys Acta 1839: 1097-1109, 2014.

9. Lee NH and Saeed AI: Microarrays. In: Protocols for Nucleic Acid Analysis by Nonradioactive Probes. Hilario E and Mackay JF (eds). Vol 353. Humana Press, New York, NY, pp265-300, 2007.

10. Butte A: The use and analysis of microarray data. Nat Rev Drug Discov 1: 951-960, 2002.

11. Edgar R, Domrachev M and Lash AE: Gene expression omnibus: NCBI gene expression and hybridization array data repository. Nucleic Acids Res 30: 207-210, 2002.

12. Barrett T, Troup DB, Wilhite SE, Ledoux P, Rudnev D, Evangelista C, Kim IF, Soboleva A, Tomashevsky M and Edgar R: NCBI GEO: Mining tens of millions of expression profiles-database and tools update. Nucleic Acids Res 35: D760-D765, 2007.

13. Salmena L, Poliseno L, Tay Y, Kats L and Pandolfi PP: A ceRNA hypothesis: The rosetta stone of a hidden RNA language? Cell 146: 353-358, 2011.

14. Tay Y, Rinn J and Pandolfi PP: The multilayered complexity of ceRNA crosstalk and competition. Nature 505: 344-352, 2014.

15. Rosenbloom KR, Armstrong J, Barber GP, Casper J, Clawson H, Diekhans M, Dreszer TR, Fujita PA, Guruvadoo L and Haeussler M: The UCSC genome browser database: 2015 update. Nucleic Acids Res 43: D670-D681, 2015.

16. Liu C, Bai B, Skogerbø G, Cai L, Deng W, Zhang, Bu D, Zhao Y and Chen R: NONCODE: An integrated knowledge database of non-coding RNAs. Nucleic Acids Res 33: D112-D115, 2005.

17. Ritchie ME, Phipson B, Wu D, Hu Y, Law CW, Shi W and Smyth GK: Limma powers differential expression analyses for RNA-sequencing and microarray studies. Nucleic Acids Res 43: e47, 2015 .
18. Reczko M, Maragkakis M, Alexiou P, Grosse I and Hatzigeorgiou AG: Functional microRNA targets in protein coding sequences. Bioinformatics 28: 771-776, 2012.

19. Betel D, Koppal A, Agius P, Sander C and Leslie C: Comprehensive modeling of microRNA targets predicts functional non-conserved and non-canonical sites. Genome Biol 11: R90, 2010.

20. Wang X: miRDB: A microRNA target prediction and functional annotation database with a wiki interface. RNA 14: 1012-1017, 2008.

21. Kertesz M, Iovino N, Unnerstall U, Gaul U and Segal E: The role of site accessibility in microRNA target recognition. Nat Genet 39: 1278-1284, 2007.

22. Lewis BP, Burge CB and Bartel DP: Conserved seed pairing, often flanked by adenosines, indicates that thousands of human genes are microRNA targets. Cell 120: 15-20, 2005.

23. Dweep H, Sticht C, Pandey P and Gretz N: miRWalk-database: Prediction of possible miRNA binding sites by 'walking' the genes of three genomes. J Biomed Inform 44: 839-847, 2011.

24. Xiao F, Zuo Z, Cai G, Kang S, Gao X and Li T: miRecords: An integrated resource for microRNA-target interactions. Nucleic Acids Res 37: D105-D110, 2009.

25. Shannon P, Markiel A, Ozier O, Baliga NS, Wang JT, Ramage D, Amin N, Schwikowski B and Ideker T: Cytoscape: A software environment for integrated models of biomolecular interaction networks. Genome Res 13: 2498-2504, 2003.

26. Kanehisa M and Goto S: KEGG: Kyoto encyclopedia of genes and genomes. Nucleic Acids Res 28: 27-30, 2000.

27. Cao C, Sun J, Zhang D, Guo X, Xie L, Li X, Wu D and Liu L: The long intergenic noncoding RNA UFC1, a target of microRNA 34a, interacts with the mRNA stabilizing protein HuR to increase levels of $\beta$-catenin in HCC cells. Gastroenterology 148: 415-426 e418, 2015.

28. Chen J, Fu Z, Ji C, Gu P, Xu P, Yu N, Kan Y, Wu X, Shen R and Shen Y: Systematic gene microarray analysis of the lncRNA expression profiles in human uterine cervix carcinoma. Biomed Pharmacother 72: 83-90, 2015.

29. Yang F, Zhang L, Huo XS, Yuan JH, Xu D, Yuan SX, Zhu N, Zhou WP, Yang GS, Wang YZ, et al: Long noncoding RNA high expression in hepatocellular carcinoma facilitates tumor growth through enhancer of zeste homolog 2 in humans. Hepatology 54: 1679-1689, 2011.

30. Kohl M, Wiese S and Warscheid B: Cytoscape: Software for visualization and analysis of biological networks. Methods Mol Biol 696: 291-303, 2011.

31. Livak KJ and Schmittgen TD: Analysis of relative gene expression data using real-time quantitative PCR and the 2(-Delta Delta C(T)) method. Methods 25: 402-408, 2001.

32. Wong QW, Ching AK, Chan AW, Choy KW, To KF, Lai PB and Wong N: MiR-222 overexpression confers cell migratory advantages in hepatocellular carcinoma through enhancing AKT signaling. Clin Cancer Res 16: 867-875, 2010.

33. Goto Y,Kojima S, Nishikawa R, Kurozumi A, Kato M, Enokida H, Matsushita R, Yamazaki K, Ishida Y, Nakagawa M, et al: MicroRNA expression signature of castration-resistant prostate cancer: The microRNA-221/222 cluster functions as a tumour suppressor and disease progression marker. Br J Cancer 113: 1055-1065, 2015.

34. Zhang Y, Takahashi S, Tasaka A, Yoshima T, Ochi $\mathrm{H}$ and Chayama K: Involvement of microRNA-224 in cell proliferation, migration, invasion and anti-apoptosis in hepatocellular carcinoma. J Gastroenterol Hepatol 28: 565-575, 2013.

35. Bi Q, Tang S, Xia L, Du R, Fan R, Gao L, Jin J, Liang S, Chen Z, $\mathrm{Xu}$ G, et al: Ectopic expression of MiR-125a inhibits the proliferation and metastasis of hepatocellular carcinoma by targeting MMP11 and VEGF. PLoS One 7: e40169, 2012.

36. Ding J, Huang S, Wang Y, Tian Q, Zha R, Shi H, Wang Q, Ge C, Chen T, Zhao Y, et al: Genome-wide screening reveals that miR-195 targets the TNF- $\alpha / N F-\kappa B$ pathway by down-regulating $\mathrm{I} \kappa \mathrm{B}$ kinase alpha and TAB3 in hepatocellular carcinoma. Hepatology 58: 654-666, 2013.

37. Yang X, Yu J, Yin J, Xiang Q, Tang H and Lei X: MiR-195 regulates cell apoptosis of human hepatocellular carcinoma cells by targeting LATS2. Pharmazie 67: 645-651, 2012.

38. Um SH, Mulhall C, Alisa A, Ives AR, Karani J, Williams R, Bertoletti A and Behboudi S: Alpha-fetoprotein impairs APC function and induces their apoptosis. J Immunol 173: 1772-1778, 2004. 
39. Duvoux C, Roudotâ-'Thoraval F, Decaens T, Pessione F, Badran H, Piardi T, Francoz C, Compagnon P, Vanlemmens C, Dumortier J,et al: Liver transplantation for hepatocellular carcinoma: A model including $\alpha$-fetoprotein improves the performance of Milan criteria. Gastroenterology 143: 986-994. e3, 2012.

40. Xue Y, Bowen B, Orr A, Koral K, Haynes M, Bell A, Paranjpe S, Mars W and Michalopoulos G: GPC3-CD81 axis in the HCV mediated liver carcinogenesis. FASEB J 29: 611-619, 2015.

41. Lei CJ, Yao C, Pan QY, Long HC, Li L, Zheng SP, Zeng C and Huang JB: Lentivirus vectors construction of SiRNA targeting interference GPC3 gene and its biological effects on liver cancer cell lines Huh-7. Asian Pac J Trop Med 7: 780-786, 2014.

42. Koo CY, Muir KW and Lam EW: FOXM1: From cancer initiation to progression and treatment. Biochim Biophys Acta 1819: 28-37, 2012 .

43. Zhao J, Liu Y, Zhang W, Zhou Z, Wu J, Cui P, Zhang Y and Huang G: Long non-coding RNA Linc00152 is involved in cell cycle arrest, apoptosis, epithelial to mesenchymal transition, cell migration and invasion in gastric cancer. Cell Cycle 14: 3112-3123, 2015

44. Pickard M, Mourtada-Maarabouni M and Williams G: Long non-coding RNA GAS5 regulates apoptosis in prostate cancer cell lines. Biochim Biophys Acta 1832: 1613-1623, 2013.
45. Xu C, Shao Y, Xia T, Yang Y, Dai J, Luo L, Zhang X, Sun W, Song H, Xiao B and Guo J: lncRNA-AC130710 targeting by miR-129-5p is upregulated in gastric cancer and associates with poor prognosis. Tumour Biol 35: 9701-9706, 2014.

46. Braconi C, Kogure T, Valeri N, Huang N, Nuovo G, Costinean S, Negrini M, Miotto E, Croce CM and Patel T: microRNA-29 can regulate expression of the long non-coding RNA gene MEG3 in hepatocellular cancer. Oncogene 30: 4750-4756, 2011.

This work is licensed under a Creative Commons Attribution-NonCommercial-NoDerivatives 4.0 International (CC BY-NC-ND 4.0) License. 